\title{
MITTEILUNGEN
}

\section{Die Rolle der Demoskopie in der Politik - Demoskopie als politischer Einflussfaktor? Eine Veranstaltung der DVParl am 12. Juni 2013 in Berlin}

Die wechselseitige Kommunikation zwischen Bürgern und Politikern ist ein zentraler Bestandteil der repräsentativen Demokratie. Neben dem direkten Kontakt der Repräsentanten zu den Repräsentierten bietet die Demoskopie ein hilfreiches Instrumentarium zur Ermittlung von Einstellungen, Stimmungen oder Wünschen der Bevölkerung. Die gleichzeitige Kritik an der Meinungsforschung ist dabei so alt wie das Instrumentarium selbst: Misst sie nur, oder nimmt sie auch Einfluss? Fest steht: Die Ergebnisse der Umfrageinstitute sind aus dem politischen Leben nicht mehr wegzudenken, vor allem dann, wenn Wahlen anstehen. Dies war Anlass genug für die Deutsche Vereinigung für Parlamentsfragen (DVParl), sich vor der diesjährigen Bundestagswahl in einer Abendveranstaltung am 12. Juni 2013 mit dem Thema „Die Rolle der Demoskopie in der Politik - Demoskopie als politischer Einflussfaktor?" zu befassen. Unter der Moderation von Heinrich Oberreuter diskutierten Richard Hilmer (Infratest dimap), Matthias Machnig (Minister für Wirtschaft, Arbeit und Technologie des Freistaates Thüringen) und Nico Fried (Parlamentsredaktion der Süddeutschen Zeitung) das Thema aus drei unterschiedlichen Blickwinkeln.

Richard Hilmer, der den Auftakt machte, wies auf die veränderte Auftragslage der Demoskopie hin: Ließen früher vor allem die Parteien Stimmungen zu ihrer Politik erheben, geben heute überwiegend die Medien Umfragen in Auftrag. Dadurch werden die Ergebnisse regelmäßig publiziert. Die vielfältigen Umfrageinstrumente, die nicht ausschließlich in der Sonntagsfrage aufgehen, beeinflussen dabei politische Akteure stärker als die Bürger. Diesen Zusammenhang veranschaulichte Hilmer differenziert an der Niedersachsenwahl 2013: Nicht die beständig niedrigen Umfragewerte der FDP deutlich unterhalb der Fünfprozenthürde veranlassten die Bürger verstärkt, die Liberalen zu wählen, sondern die von der CDU lancierte Leihstimmenkampagne zugunsten des Koalitionspartners. Damit stellte er sich gegen die These, die veröffentlichten niedrigen Umfragewerte der FDP hätten die Bürger bei ihrer Wahl beeinflusst. Hilmer sieht damit die Demoskopie lediglich als Hilfsinstrument zur Meinungsbildung.

Die Perspektive des wahlkampferprobten Politikers brachte Matthias Machnig ein, der die erfolgreichen Wahlkämpfe der SPD in den Jahren 1998 und 2002 leitete. Er unterteilt drei Facetten der Demoskopie: (1) Die Umfragen bilden eine politische Stimmung ab, nicht aber ein Wahlergebnis. Sie sind somit eine Orientierungshilfe, dienen jedoch nicht als Prognose. (2) Die Umfragen können auch hilfreich in der Justierung der politischen Richtung sein, indem sie aufzeigen, welche Themen Wähler wie ansprechen. (3) Die erhobenen Einstellungen und Stimmungen nützen der zwischen- und innerparteilichen Auseinandersetzung. Richtig ausgewertet sind sie hilfreich bei der inhaltlichen Ausrichtung der Parteiprogramme und Wahlkämpfen.

Im Vergleich zu Werbekampagnen in der Wirtschaft oder auch zu US-amerikanischen Wahlkämpfen kritisierte Machnig die fehlende Professionalisierung in Deutschland. Die 
Ergebnisse der Meinungsforschung finden seiner Auffassung nach zu wenig Berücksichtigung in den Planungen von Wahlkämpfen. Gleichzeitig wies er aber auch mit Blick auf Unentschlossene und Nichtwähler darauf hin, dass mit den erhobenen Daten vorsichtig umzugehen ist. Diese Gruppe wird in den Umfragen nicht aufgeführt: „Es wird aber so getan und in den Umfragen eingepreist, als würden diejenigen, die nicht entschieden sind, gar nicht zur Wahl gehen."

Nico Fried stellte als abschließender Redner den Umgang der Medien und Journalisten mit Umfragen und die Bedeutung für den politikjournalistischen Alltag in den Mittelpunkt seines Referates. Auch er beobachtet einen großen Einfluss der Demoskopie auf die Politik. Am Beispiel der Bundestagwahl 2005 zeigte er, „wie unkritisch Medien und Journalisten Umfragezahlen übernommen haben“, als sie sich bereits vor dem Wahltermin festlegten, dass Schwarz-Gelb keine Mehrheit erzielen würde. Die Ergebnisse der Meinungsforschung sollten dagegen vor allem zur Unterstützung der Analyse verwendet werden, die nicht an die Umfrageinstitute ausgelagert werden sollte.

Auch Fried nahm das Beispiel der Landtagswahl in Niedersachsen 2013 kritisch auf: Da Wahlentscheidungen auf Grund letzter Umfrageergebnisse auch erst am Tag der Wahl zustande kommen können, ist es den Demoskopen nur schwer möglich, strategisches Verhalten der Wähler zu berücksichtigen. Somit ermöglichten die dauerhaft niedrigen Umfragewerte der FDP, so Fried, erst ihr überraschend gutes Ergebnis.

Bei der anschließenden Diskussion standen zwei Themen im Vordergrund. Niels Diederich (Freie Universität Berlin) fragte, warum der Anteil der unentschiedenen Wähler von den Umfrageinstituten in der Regel nicht veröffentlicht wird. In den Ergebnissen der Sonntagsfrage sind die unentschlossenen Wähler aus der Statistik herausgerechnet. Hilmer erwiderte, dass diese Zahlen zwar erhoben werden, aber schwer zu interpretieren sind. Fried fügte hinzu, dass das Wissen um die Zahl der Unentschlossenen wenig über deren Verhalten am Tag der Wahl aussagt. Daher wird die Auswertung nicht regelmäßig vorgenommen.

Ute Vogt lenkte die Diskussion auf ein anderes Thema: Die Wähler in Niedersachsen hätten gemäß ihrer Parteipräferenz entschieden, sofern Umfrageergebnisse den Einzug der Liberalen in den Landtag nicht in Frage gestellt hätten. Sie sprach sich dafür aus, Umfragen ab vierzehn Tage vor einer Wahl nicht mehr zu veröffentlichen. Dies sah Hilmer kritisch. Für ihn ist es ein Recht des Bürgers, Umfragedaten auch direkt vor einer Wahl als Orientierungshilfe zu nutzen. Letztlich sei der größere Einfluss der Parteien auf den Wähler entscheidend. Auch Fried stellte sich gegen diese Forderung. Würde der Wähler tatsächlich die veröffentlichten Zahlen als Referenz nutzen, richtete er sich eben an zwei Wochen alten Zahlen aus. Am Grundproblem ändere dies nichts.

Insgesamt bot der Abend interessante Vorträge und eine lebhafte Diskussion. Es bleibt vor allem, dass es nicht nur auf die Sonntagsfrage ankommt, sondern Meinungsumfragen auch als (erkenntnisförderndes) Planungsinstrument für inner- und zwischenparteiliche Differenzen eingesetzt werden. Zu bedenken bleibt immer, dass repräsentative Demokratie sich nicht allein an einer durch Demoskopen ermittelten (messbaren) Mehrheitsmeinung ausrichten kann. Sie besteht vielmehr aus Responsivität und politischer Führung. 